\title{
THE Ha/HB RATIO IN SOLAR FLARES
}

\author{
HAROLD ZIRIN, MARGARET LIGGETT, and ALAN PATTERSON \\ Big Bear Solar Observatory, California Institute of Technology, Calif., U.S.A.
}

(Received 7 November, 1980; in revised form 16 April, 1981)

Abstract. We have measured the ratio of $\mathrm{H} \alpha$ to $\mathrm{H} \beta$ central intensities in the peak kernels of 14 flares, using simultaneous filtergrams. The ratio is typically one with some scatter. By contrast, in stellar flares the ratio is about 0.8 .

In recent years we have seen Zirin (1978) that the intensity ratio of hydrogen lines is not always what we expect it to be. Important physical information can be obtained from these ratios. Most attention lately has been given to the $\mathrm{H} \alpha / \mathrm{L} \alpha$ ratio, but one should be able to learn from Balmer ratios as well. In stellar flares, for example, it has been found (Kunkel, 1970; Mochnacki and Zirin, 1980) that $\mathrm{H} \gamma$ is the most intense Balmer line; this is attributed to the high temperature of the stellar flares. Thus in these events we have a Balmer increment for $n<6$.

There is little published data on the $\mathrm{H} \alpha / \mathrm{H} \beta$ ratio in solar flares. The most complete data are given by Smith (1963) on the great flare of September 2, 1960. Smith found central intensity (relative to local continuum) ratios of $1.13,0.848$, and 1.27 in start, maximum and postmaximum flare stages, respectively. These ratios must be corrected for the $6563 / 4861$ continuum ratio and also corrected to the frequency scale by dividing by $\lambda^{2}$; an overall factor 1.18 is obtained, giving $1.33,1.0$, and 1.50 , for the $\mathrm{H} \alpha / \mathrm{H} \beta$ ratios of $F_{\nu}$ at start, max and decline. Smith gives line widths to zero intensity for the Balmer lines; the widths for $\mathrm{H} \alpha$ and $\mathrm{H} \beta$ are equal, which means that the $\mathrm{H} \beta$ width in the frequency scale is 1.8 times greater than that for $\mathrm{H} \alpha$, and the integrated intensity correspondingly greater. Jefferies $e t$ al. (1959) give similar widths, but give no intensities. Smith found $\mathrm{H} \alpha / \mathrm{H} \beta$ (central intensity $)=1.13,0.848$, and 1.27 at the start, peak, and decay of the flare. Reduced to $F_{\nu}$, these ratios are in good agreement, with $\mathrm{H} \beta$ about 1.4 stronger than $\mathrm{H} \alpha$. We have used Smith's central intensities rather than the intensity above background, because the flare emission is obviously optically deep. The stellar flare data has subtracted the continuum, because we do not know the relative areas of line and continuum sources; the possible error introduced this way is only significant for the post flare measurements. Note that although the half-widths given by Smith decrease in the higher Balmer lines, the corrected widths $d \lambda / \lambda$ increase.

Švestka (1960) measured $H \alpha$ and $H \beta$ simultaneously in a flare and found $H \alpha$ 3 times the local continuum and $\mathrm{H} \beta 4$ times the local continuum, giving a ratio of $F_{\nu}$ of 1.5 . Švestka (1976) gives line width data from himself and Suemoto and Hiei indicating a half-width ratio of 1.3 corresponding to constant $d \lambda / \lambda$. The consensus of these data appears to be that $\mathrm{d} \lambda / \lambda$ for $\mathrm{H} \beta$ is equal to or greater than that for $\mathrm{H} \alpha$, a remarkable and hitherto unnoticed result. 
It is most difficult to obtain spectra of the flash phase of flares; these data were obtained by heroic efforts. But we do not know the exact slit locations in the flare, or other parameters (although the great half width obtained by Smith suggests she was at the right place). The great difficulty in obtaining scanning spectra of flares in both wavelengths makes it impossible to obtain a representative sample.

In the present work we have studied an extensive body of data accumulated at Big Bear of simultaneous $\mathrm{H} \alpha$ and $\mathrm{H} \beta$ cinematography of flares. The data are obtained with two telescopes simultaneously photographing flares in $\mathrm{H} \alpha$ and $\mathrm{H} \beta$; $\mathrm{H} \alpha$, with a Zeiss $\frac{1}{4} \AA$ filter on our $25 \mathrm{~cm}$ refractor, and $\mathrm{H} \beta$ with the $65 \mathrm{~cm}$ Gregorian and a Zeiss universal filter, band pass about $0.18 \AA$ at $\mathrm{H} \beta$. Most of the time the $65 \mathrm{~cm}$ was operated with a minifying system, so the scales are not too different. Figure 1 shows $\mathrm{H} \alpha$ and $\mathrm{H} \beta$ filtergrams of two flares in 1979. The November 5 flare was an impulsive large flare, class 2, while the May 17 flare was smaller. The appearance of the active regions is different, although one may at once ascertain from the arch structures that both are Balmer lines. In $\mathrm{H} \beta$ plages are hardly visible, surrounding faculae are faint, but sunspots are prominent. All this means that $\mathrm{H} \beta$ has lower optical depth than $\mathrm{H} \alpha$. In the flares we see that while the kernels in $\mathrm{H} \alpha$ and $\mathrm{H} \beta$ are similar, outlying parts of the flares are much fainter in $H \beta$, again probably a consequence of lower optical depth in $\mathrm{H} \beta$. Our measurements were only made in the kernels of the flares, and we only measured the intensity at line center. While it would be desirable to obtain integrated intensities also for comparison with stellar data, the ratio of central intensities is an important test of models of Balmer line formation in flares. We also use some of the published line width observations to estimate the ratios of integrated intensity.

Fourteen flares were chosen for which good $\mathrm{H} \alpha$ and $\mathrm{H} \beta$ filtergrams were obtained within a few seconds of each other. In each case the intensity of the peak flare emission was measured with a $35 \mathrm{~mm}$ overhead projector and a suitably masked photodiode; intensities relative to the quiet chromosphere were measured by comparison with the calibration wedge developed with each film. No line width information is obtained, only the peak intensity could be measured. Integrated line measurements may now be made with computer control of the filter and will be obtained this year. The filtergrams show the spatial distribution, hence the most intense portion of the flare was easily picked out and measured with a 1 arc sec aperture.

The ratio of $\mathrm{H} \alpha$ and $\mathrm{H} \beta$ intensity to the chromosphere being known, the relative intensity of the two lines could be determined. We first determined the centerline/continuum ratio for the photosphere by taking filtergrams of the sun center both in centerline and a clear point in the nearby continuum (we used $\mathrm{H} \alpha-13.22 \AA$ and $\mathrm{H} \beta-10.5 \AA$ ) with the same exposure interval, but a series of calibrated neutral filters inserted for the continuum measurement. We found the line/continuum ratios to be 0.33 for $\mathrm{H} \alpha$ and 0.313 for $\mathrm{H} \beta$; these values are greater than the true central intensity of these lines because of side bands in the filter as well as the finite bandwidth. The peak intensities were then intercompared using the known spectrum of the Sun. We made no further correction for differential limb darkening, 


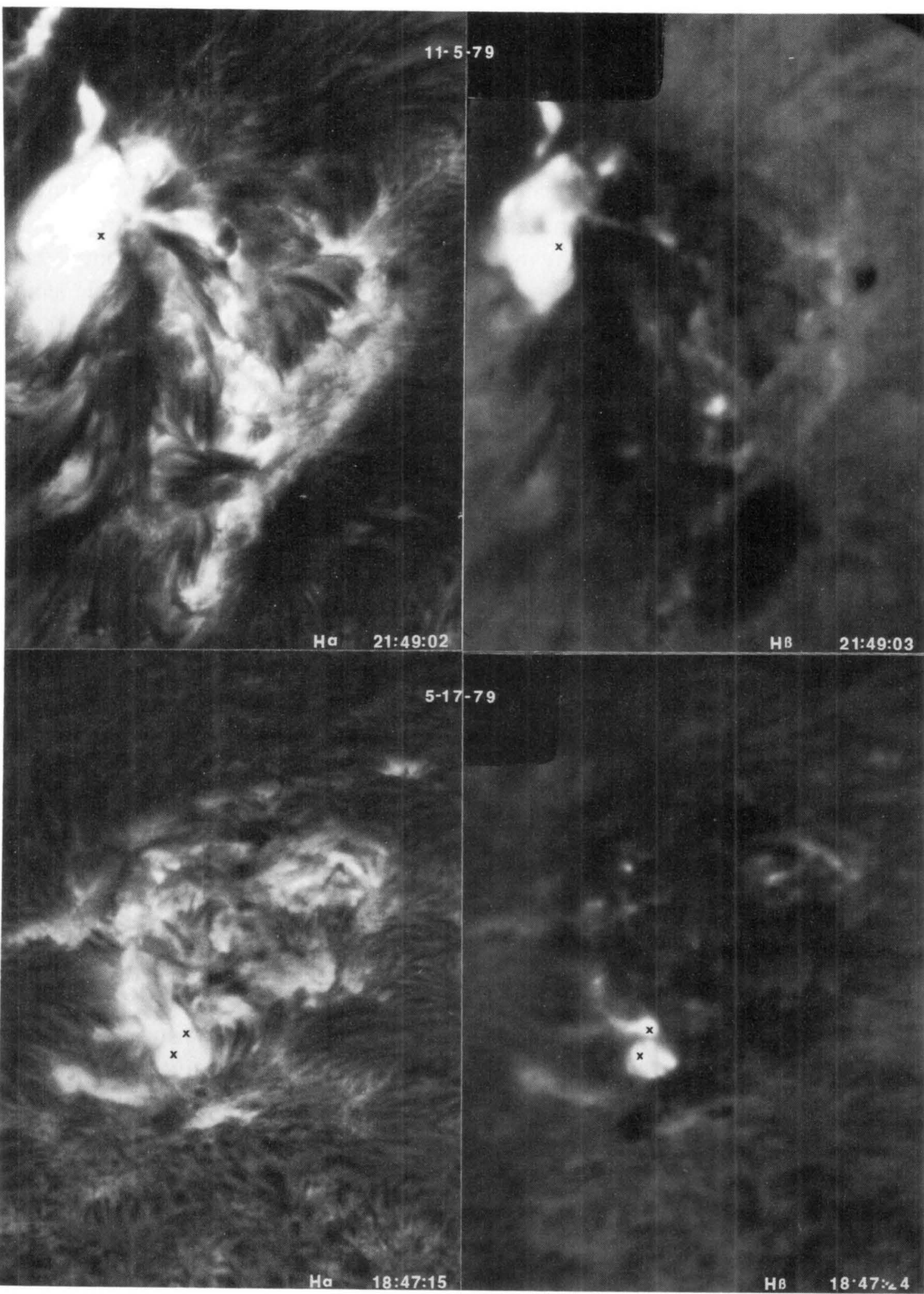

Fig. 1. Filtergrams of two flares in $\mathrm{H} \alpha$ and $\mathrm{H} \beta$. The November 5, 1979 flare was an impulsive class 2 event, while the May 1-7 flare was smaller. The appearance of the active regions is different, although one may at once ascertain from the arch structures that both are Balmer lines. In $\mathrm{H} \beta$ plages are hardly visible, surrounding faculae are faint, but sunspots are prominent. All this means that $\mathrm{H} \beta$ has lower optical depth that $\mathrm{H} \alpha$. In the flares we see that while the kernels in $\mathrm{H} \alpha$ and $\mathrm{H} \beta$ are similar, outlying parts of the flares are much fainter in $\mathrm{H} \beta$, again probably a consequence of lower optical depth in $\mathrm{H} \beta$.

Our measurements were only made in the kernels of the flares. 
which should be small. We also assume the band pass of the filter does not change between the filter and continuum setting. This was verified by examing the filter bandpass on the spectrograph.

We do not have a photometric record of the bandpass of the filter; however we do know, as mentioned above, that the measured intensity at line center is about 0.3 for each filter. If the true central intensity of $\mathrm{H} \alpha$ is about 0.2 , then a continuum contribution of about $0.1 \times$ continuum must be present, coming both from near and far side bands. This is confirmed by the presence of a 'second limb' on chromosphere frames. However the flare profiles are quite broad compared to the near side bands, and do not introduce any sizeable error. The method of calibration excludes any error due to broad band spurious light because we use the chromosphere cum scattered light as an intermediate standard only, which is then referred, in a consistent way, to the continuum. If the $\mathrm{H} \alpha / \mathrm{H} \beta$ ratio were large, this contribution could introduce some error; but since the ratio is close to unity, the error introduced by spurious light must be quite small.

Since the lines appear to be optically thick we have not subtracted the background intensity; we believe that would give erroneous values. However the background intensity can be removed by simply subtracting unity from the flare/chromosphere ratio given as all the flares studied here were seen against the chromospheric background.

Table I gives the results of measurements in a number of flares. The flares were selected purely by optical quality of the data. The fourth column gives the intensity relative to the chromosphere for $\mathrm{H} \alpha$; the fifth, the ratio of the raw intensities relative to the local chromosphere. The sixth column gives the ratio of central intensities Fnu in the frequency scale; the seventh gives the ratio of integrated intensities for $\mathrm{d} \lambda / \lambda=\mathrm{const}$. (corresponding to the results of Švestka and of Suemoto and Hiei), and the last, the ratio of integrated intensities for constant $\mathrm{d} \lambda$ (corresponding to the result of Elske Smith). The ratio of $F_{\nu}$ is important for comparison with the Planck function, but integrated intensities must be compared with the stellar data.

Table I shows that on the average, Fnu for the central intensity of $\mathrm{H} \alpha$ is greater than that for $\mathrm{H} \boldsymbol{\beta}$; the average ratio is about 1.3 , so there is no great contradiction with expected results, although the data do disagree with Smith's result. However if we use either assumption for the line width of $\mathrm{H} \alpha$ and $\mathrm{H} \beta$ we get a ratio less than unity, somewhat similar to the stellar flare data. We find no particular correlation of the line ratios with flare intensity; ratios near unity can be found for the brightest and weakest flares, and ratios up to 1.5 also. We have not measured the intensity in the decay phase; visually it appears to behave like stellar flares, $\mathrm{H} \alpha$ decaying more slowly and thus becoming relatively more intense.

The stellar data are of course integrated over flare area and profile. We have not integrated over area, and it is clear from the illustration that $\mathrm{H} \alpha$, being more extensive, would benefit from such an integration. However, the centerline frames distort the situation. As Zirin and Tanaka (1973) showed, most of the H $\alpha$ energy 
TABLE I

The results of measurements in a number of flares

\begin{tabular}{|c|c|c|c|c|c|c|c|}
\hline Date & \multicolumn{2}{|l|}{ Time } & $\frac{\mathrm{H} \alpha \text { flare }}{\text { chromo }}$ & $\frac{\mathrm{H} \alpha \text { raw }}{\mathrm{H} \beta \text { raw }}$ & $\frac{F \nu(\alpha)}{F \nu(\beta)}$ & $\frac{F_{\nu} \text { (integral) }}{\text { const. } \Delta \nu / \nu}$ & const. $d \lambda$ \\
\hline (day/month/ye & & & & & & & \\
\hline $6 / 22 / 78$ & $20: 70$ & Avg. 3 pts. & 4.10 & 1.47 & 1.73 & 1.23 & 0.95 \\
\hline $9 / 3 / 78$ & $23: 55$ & $\begin{array}{l}\text { Point A } \\
\text { Point B }\end{array}$ & $\begin{array}{l}4.08 \\
3.67\end{array}$ & $\begin{array}{l}2.37 \\
2.11\end{array}$ & $\begin{array}{l}2.8 \\
2.49\end{array}$ & $\begin{array}{l}2.07 \\
1.84\end{array}$ & $\begin{array}{l}1.53 \\
1.36\end{array}$ \\
\hline $5 / 24 / 79$ & $18: 14$ & Point $\mathrm{A}$ & 3.56 & 1.89 & 2.2 & 1.65 & 1.22 \\
\hline $\begin{array}{l}1 / 24 / 80 \\
\text { 'homologous' }\end{array}$ & $\begin{array}{l}20: 48 \\
22: 56\end{array}$ & $\begin{array}{l}\text { Point A } \\
\text { Point B } \\
\text { Point C }\end{array}$ & $\begin{array}{l}2.10 \\
2.04 \\
2.42\end{array}$ & $\begin{array}{l}1.25 \\
2.29 \\
0.74\end{array}$ & $\begin{array}{l}1.48 \\
2.29 \\
0.87\end{array}$ & $\begin{array}{l}1.09 \\
2.27 \\
0.64\end{array}$ & $\begin{array}{l}0.8 \\
1.48 \\
0.48\end{array}$ \\
\hline $1 / 25 / 80$ & $21: 10$ & Avg. 5 pts. & 3.7 & 1.03 & 1.21 & 0.90 & 0.67 \\
\hline $2 / 2 / 80$ & $17: 14$ & $\begin{array}{l}\text { Avg. } 3 \text { 'hi' pts. } \\
\text { Avg. } 3 \text { 'lo' pts. }\end{array}$ & $\begin{array}{l}2.97 \\
2.19\end{array}$ & $\begin{array}{l}1.43 \\
1.30\end{array}$ & $\begin{array}{l}1.69 \\
1.54\end{array}$ & $\begin{array}{l}1.25 \\
1.14\end{array}$ & $\begin{array}{l}0.92 \\
0.84\end{array}$ \\
\hline $2 / 4 / 80$ & $20: 47$ & Avg. 2 pts. & 2.45 & 1.5 & 1.77 & 1.31 & 0.97 \\
\hline $2 / 7 / 80$ & $00: 09$ & Avg. 5 pts. & 1.96 & 1.35 & 1.59 & 1.18 & 0.87 \\
\hline $2 / 7 / 80$ & $21: 57$ & Point A & 2.43 & 1.18 & 1.39 & 1.03 & 0.76 \\
\hline $\begin{array}{l}5 / 17 / 80 \\
\text { 'homologous } \\
\text { flare max }\end{array}$ & $\begin{array}{r}-17: 50 \\
-18: 46 \\
18: 47 \\
18: 50\end{array}$ & $\begin{array}{l}\text { Avg. } 4 \text { pts. } \\
\text { Avg. } 6 \text { pts. } \\
\text { Avg. } 6 \text { pts. } \\
\text { Avg. } 6 \text { pts. }\end{array}$ & $\begin{array}{l}1.55 \\
2.01 \\
2.40 \\
2.19\end{array}$ & $\begin{array}{l}1.11 \\
1.31 \\
1.24 \\
1.30\end{array}$ & $\begin{array}{l}1.32 \\
1.54 \\
1.46 \\
1.54\end{array}$ & $\begin{array}{l}0.98 \\
1.14 \\
1.08 \\
1.14\end{array}$ & $\begin{array}{l}0.72 \\
0.84 \\
0.80 \\
0.84\end{array}$ \\
\hline $5 / 17 / 80$ & $18: 41$ & $\begin{array}{l}\text { Bright Plage } \\
\text { Average Plage }\end{array}$ & $\begin{array}{l}1.91 \\
1.14\end{array}$ & $\begin{array}{l}1.12 \\
1.04\end{array}$ & $\begin{array}{l}1.32 \\
1.23\end{array}$ & $\begin{array}{l}0.98 \\
0.91\end{array}$ & $\begin{array}{l}0.72 \\
0.68\end{array}$ \\
\hline
\end{tabular}

is radiated in the kernels of the flare, where the profile is broad and intense for both $\mathrm{H} \alpha$ and $\mathrm{H} \beta$. So the error introduced by our procedure is not great.

If one examines the line ratios from the point of view of black body emitters, there is no great contradiction; at $6000 \mathrm{deg}$ the ratio $F_{\nu}(\alpha) / F_{\nu}(\beta)$ is 1.5 , and the emission is of course equal to the photosphere continuum, essentially as observed. The great contradiction, of course, is with the $L \alpha$ intensity; it is a fact (Zirin, 1978) that $\mathrm{H} \alpha$ intensity (in flares as well as in quasars) equals that of $\mathrm{L} \alpha$ and one explanation of this is thermalization at $18000 \mathrm{deg}$; actually the $\mathrm{H} \alpha / \mathrm{H} \beta$ ratio of $F_{\nu}$ varies little with temperature (it would be 0.7 at $18000 \mathrm{deg}$ ), but if the radiation temperature of $\mathrm{H} \alpha$ were $18000 \mathrm{deg}$, the line intensity would be $15 \times$ the photosphere, which it is not. The recent work of Canfield et al. (1981) explains the $\mathrm{L} \alpha / \mathrm{H} \alpha$ ratio at a temperature of $10000 \mathrm{deg}$ but does not address the Balmer ratios.

The the measured ratios are not too different from those in stellar flares is suggested by the last two columns of TableI which show that a variety of possible line width ratios could give an integrated intensity ratio of less than unity, as do the Smith results mentioned above, even though the central intensity of $\mathrm{H} \alpha$ is greater than that of $\mathrm{H} \beta$. 


\section{Acknowledgement}

This work was supported by NASA under grant NGL 05-002-034 and the Atmospheric Sciences section of the NSF under ATM76-21132.

\section{References}

Canfield, R. C., Puetter, R. C., and Richiazzi, P. J.: 1980, Astrophys. J. 239, 1036.

Jeffereies, J. T., Smith, E. v. P., and Smith, H. J.: 1959, Astrophys. J. 129, 146.

Kunkel, W.: 1970, Astrophys. J. 161, 503.

Mochnacki. S. and Zirin, H.: 1980, Astrophys. J. 239, 127.

Smith, E. v. P.: 1963, Astrophys. J. 137, 580.

Svestka, Z.: 1960, Bull. Astron. Inst. Czech. 11, 167.

Švestka, Z.: 1976, Solar Flares, D. Reidel, Publ. Co., Dordrecht, Holland.

Zirin, H.: 1978, Astrophys. J. 222. L105.

Zirin, H. and Tanaka, K.: 1973, Solar Phys. 32, 173. 